\title{
Determination of netrin-1 levels in patients that applied to emergency department with stroke and its effects on survival for 6 months
}

\author{
Korhan Ivelik $^{1}$, Ramazan Koylu ${ }^{1,}$, Akinci Emine ${ }^{1}$, Basar Cander ${ }^{1}$, Zerrin Defne Dundar ${ }^{1}$, \\ Oznur Koylu², Haluk Gumus ${ }^{3}$
}

${ }^{1}$ Konya Training and Research Hospital, Emergency Department, Konya, Turkey

${ }^{2}$ Konya Training and Research Hospital, Biochemistry Department, Konya, Turkey

${ }^{3}$ Konya Training and Research Hospital, Neurology Department, Konya, Turkey

\author{
Email address: \\ drkoylu@yahoo.com (R. Koylu)
}

\section{To cite this article:}

Korhan Ivelik, Ramazan Koylu, Akinci Emine, Basar Cander, Zerrin Defne Dundar, Oznur Koylu, Haluk Gumus. Determination of Netrin-1 Levels in Patients that Applied to Emergency Department with Stroke and Its Effects on Survival for 6 Months. American Journal of Internal Medicine. Vol. 2, No. 6, 2014, pp. 150-155. doi: 10.11648/j.ajim.20140206.19

\begin{abstract}
Introduction: We aimed to observe whether there are differences in patients presenting to the emergency department that has stroke, determination of serum levels of netrin-1, and its impact on patient survival and determine the effect on survival and stroke patients according to the type of netrin-1 levels. Methods: The study was held in the Konya Education and Research Hospital, Emergency Department between 1st of August, 2012 and 30th of November, 2012. Patients divided into sub groups after determination of hemorrhagic and ischemic stroke. Control group was created with the patients that did not have stroke. In both groups, Netrin-1 levels were studied. Findings: Totally 131 stroke 51 control patiens were enrolled the study. $\% 13$ of the patients were with hemorrhagic where as the other $\% 87$ was ischemic stroke. In the stroke group Netrin-1 was lower than the control group ( $\mathrm{p}=0,003$ and $\mathrm{p}<0,001)$. In order to distinguish between control and case groups, related with Netrin-1 ROC curve was statistically significant 0,739 (\%95 Confidence Interval: 0,664-0,814). Best cutoff point for Netrin- 1 is determined as 728.4 . The sensitivity of Netrin- 1 at this point is $\% 71,0$; selectivity is $\% 68,6$; positive predictive value is $\% 85,3$; negative predictive value is $\% 47,9$; and finally accuracy rate was found as $\% 70,3$. Although in the hemorrhagic stroke Netrin- 1 is lower than ischemic stroke, the difference between groups was not statistically significant ( $\mathrm{p}=0,297)$. There was not statistically significant difference in terms with among the patients the median survival and die patients related with Netrin-1. Discussion: Netrin-1 precursor protein is important for neural and vascular development. Netrin-1 regulates cell migration and axon in the brain in utero. On the other hand, in the SSS damage, injured in sections the migration of adult neural stem cells is located. In determining the prognosis of stroke and mortality in the literature, many markers are used. Result: Our study is the first study on this subject in stroke patients have low levels of netrin-1. According to the type of stroke did not detect a difference in netrin-1 levels. Netrin-1 levels were not associated with mortality.
\end{abstract}

Keywords: Stroke, Netrin-1, Survival

\section{Purpose}

Aim of this study, one of the most common reason for admission to the emergency department of cerebrovascular diseases in stroke patients to determine serum levels of Netrin-1 and by following these patients for six-month period, patients with NIHSS scores and 6 months of NIHSS scores comparission and to determine the effects on patient survival of the Netrin-1 serum levels after 6 months.

\section{Introduction}

Definition of stroke, according to World Health Organization (WHO) is; which lasted more than 24 hours or even fatal, without any other reason that can be explained but vascular, quickly developed, a kind of global disorder that sometimes focal cerebral functions (1).

Acute stroke is a medical condition; acute myocardial infarction or severe trauma that requires immediate 
intervention. Hospital emergency departments, has effective role in managing potential stroke cases. Clinician's assessment, diagnostic tests, and the desired section specializing in stroke should be performed in a short time to reach. The most important point in the patient's history, it is time to onset of symptoms. On neurological examination scoring strokes are used (2). These scores only show not only the degree of neurological deficit also likely location of vessel occlusion identification, that early diagnosis and patient compliance and possible complications of the various interventions shows. Many studies of clinicians working in emergency services, thanks to this scoring system, cases of stroke have shown that properly managed (3). Neurovascular examination should be short and full. If necessary, must be supported by specific scale. National Institutes of Health Stroke Scale (NIHSS) is one thing that can be used. In addition, this scale neurology expertise can be used even by non-physicians (2). The use of this scale and bring a standard to facilitate the detection and the severity of symptoms as well as ensure that health workers are more easily negotiated (4). With this scale by doctors working in the emergency department diagnosis of stroke management is provided and maintained completely (3).

\subsection{Netrin Protein Family}

Netrins precursor protein is important for neural and vascular development (5). For mammals there are five netrin expression defined $(1,3,4, \mathrm{G} 1$ ve G2). Netrin-1, weighing 50 to $75 \mathrm{kDa}$ laminin-like protein (6). Intercellular guidance tasks, axonal growth harnesses and headed towards the target zone here is to create synapses (7). Netrin-1 deficient mice hippocampal commissure and corpus callosum agenesis, resulting in major disruptions seen in the formation of axon projections (8).

Netrin-1, is the unique therapeutic potential for angiogenic effects in pathological conditions in the regulation of blood circulation has been suggested. For example; netrin-1 is to accelerate neovascularization in experimental ischemia (9), functional aspects of the heart infarct formed sufficient to increase the density of blood vessels (10) and adenoassociated virus gene transfer associated with adult netrin-1 has been shown to cause neovascularization in rat brain (11).

\section{Materials and Methods}

We carried out this study on 131 patients who admitted to the emergency department at the first 6 hours from the beginning of the stroke symptoms, between August 1, 2012 and November 30, 2012. Control group consisted of 51 healthy human. This study is controlled, open, observational, and prospective clinical trial.

Patients and/or their relatives to provide information about the study, patients who agreed to participate were included in the study. Our first assessment of emergency department patients with suspected stroke, after physical examination, making an emergency intervention in the routine diagnosis of stroke patients with stable brain BT drawn according to the protocol primarily hemorrhagic, ischemic stroke distinction was made. Bleeding in patients without brain BT ADC map drawn by the diffusion-weighted MR hyperintense T2 hypointense areas in the presence of cross-sections of ischemic stroke was accepted. While the blood of patients examined in the emergency room, start by taking $5 \mathrm{cc}$ of blood biochemistry tube netrin-1 levels were studied.

Neurological examination of the patient NIHSS (National Institutes of Health Score Scale) score was evaluated. Accordingly, patients with NIHSS score of mild, moderate and severe neurological deficits were classified as. Patients with NIHSS scores of 15 and above severe neurological deficits, with NIHSS score 8-14 moderate neurological deficits, NIHSS score of 1-7 fields were considered mild neurological deficits.

Patients with approval, patients over 18 years of age, patients without previous stroke were included in the study. Patients who did not their approval, pregnant women, patients under 18 years of age, breast-feeding women, patients who received thrombolytic therapy, patients who underwent surgery were excluded from the study. In our study, non-stroke patients admitted to the emergency room for any reason, 50 adults over age 60 netrin levels were measured by taking blood from the control group was created.

Study performed with using Elisa kit of Cusabio brand Human netrin. (Catalog Number: CSB-E11899h). Measurements read by RAYTO brand Elisa plate reader device. Computed tomography for the study performed with the six sections of Siemens SOMATOM Emotion Earling / Germany origin device. MR Imaging is made with Siemens Somatom Avanto 1,5 tesla Earlingen/Germany origin device.

\section{Statistical Analysis}

Data analysis was performed using SPSS for Windows 11.5 package program. Whether it is appropriate to normal distribution of continuous variables was evaluated by the Shapiro-Wilk test. Descriptive statistics for continuous variables as mean \pm standard deviation or median (minimum - the largest), the categorical variables and the number of caseswas shown in the form (\%).

The difference between the groups in terms of mean values of materiality, Student's t-test of the significance of the difference in median values, when there are two independent groups with the Mann-Whitney U test, amoung more than two independent groups were analyzed with the KruskalWallis test. Kruskal-Wallis test statistic if there is a significant difference in the results in order to identify the cause of Conover's multiple comparison test was used.

Categorical variables, Pearson's chi-square or Fisher's exact Outcome was assessed by the chi-square test. Whether there is a significant correlation between continuous variables, examined with Spearman's correlation tests. In the case group, whether there is a significant difference between the initial and sixth month NIHSS levels were analyzed by Wilcoxon sign test.

To distinguish between the case and control groups, 
determining whether netrin measurements ROC analysis was assessed by calculating the area under the curve. If there is an important area under the curve of the best cut point was determined using the Youden Index. In addition, the related sensitivity, specificity, and positive and negative predictive values were calculated with accuracy.

$\mathrm{p}$ value of $<0.05$ was considered statistically significant.

\section{Findings}

Between, cases in the group of patients who were followed at the time of application level median NIHSS and 6 months follow-up results in a median NIHSS level (Table-1), no statistically significant difference was seen $(\mathrm{p}=0,096)$.

Netrin cases compared to control group median levels (Figure 1, Table-2) were statistically significantly lower $(\mathrm{p}<0,001)$.

Table 1. NIHSS Scores

\begin{tabular}{ll}
\hline Follow-up Time & NIHSS \\
\hline at the beginning & $7(0-36)$ \\
6.month & $8(0-36)$ \\
P value & 0,096 \\
\hline
\end{tabular}

Table 2. Netrin Levels

\begin{tabular}{llll}
\hline variables & Controle group $(\mathbf{n = 5 1})$ & Case group $(\mathbf{n = 1 3 1})$ & p-value \\
\hline Netrin & $788,8(209,7-2578,8)$ & $611,6(40,1-2271,9)$ & $<0,001$ \\
\hline
\end{tabular}

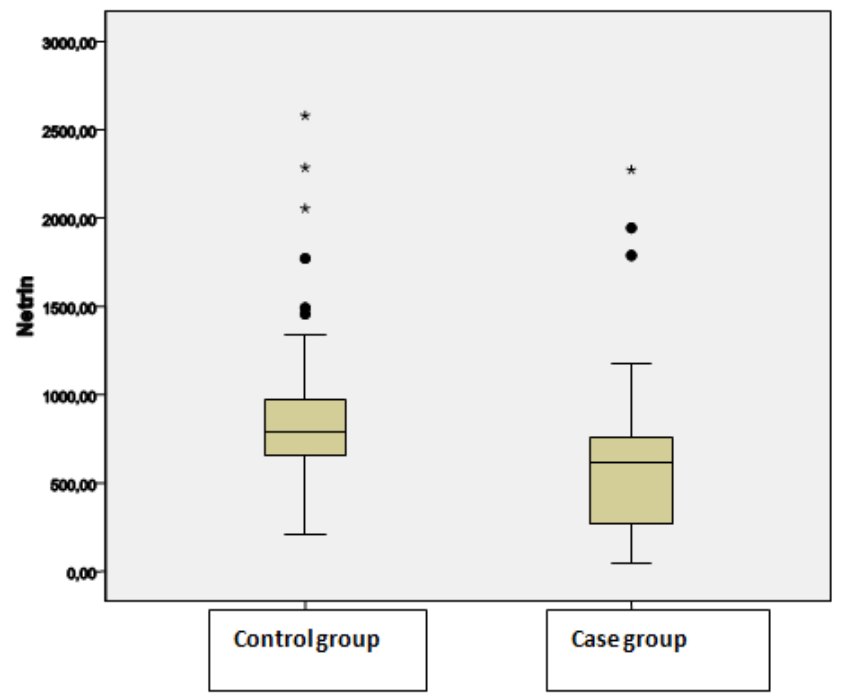

Figure 1. Distribution of netrin

To distinguish between control and case groups netrin related to the area under the ROC curve (Figure 2, Table 3) is statistically significant 0.739 (95\% confidence interval: 0.664 to 0.814$)$, respectively ( $\mathrm{p}<0,001)$.

Best cut-off point regarding to Netrin was found as 728.4 $\mathrm{pg} / \mathrm{ml}$. At this point, netrin sensitivity $71.0 \%$; specificity $68.6 \%$; positive predictive value was $85.3 \%$; negative predictive value of $47.9 \%$; and accuracy rate found as $70.3 \%$.

GP: Real Positive; YN: False Negative; GN: Real Negative; YP: False Positive; PTD: Positive Predictive Value; NTD: Negative Predictive Value.
Control, hemorrhagic stroke and ischemic stroke group median levels of netrin (Table 4), compared to the control group, respectively, hemorrhagic stroke and ischemic stroke group, the median level of netrin statistically found significantly lower $(\mathrm{p}=0.029$ and $\mathrm{p}<0.001)$.

Ischemic stroke, hemorrhagic stroke compared with the median of the group despite a lower level of netrin difference between the groups was not statistically significant $(\mathrm{p}=$ 0.297).

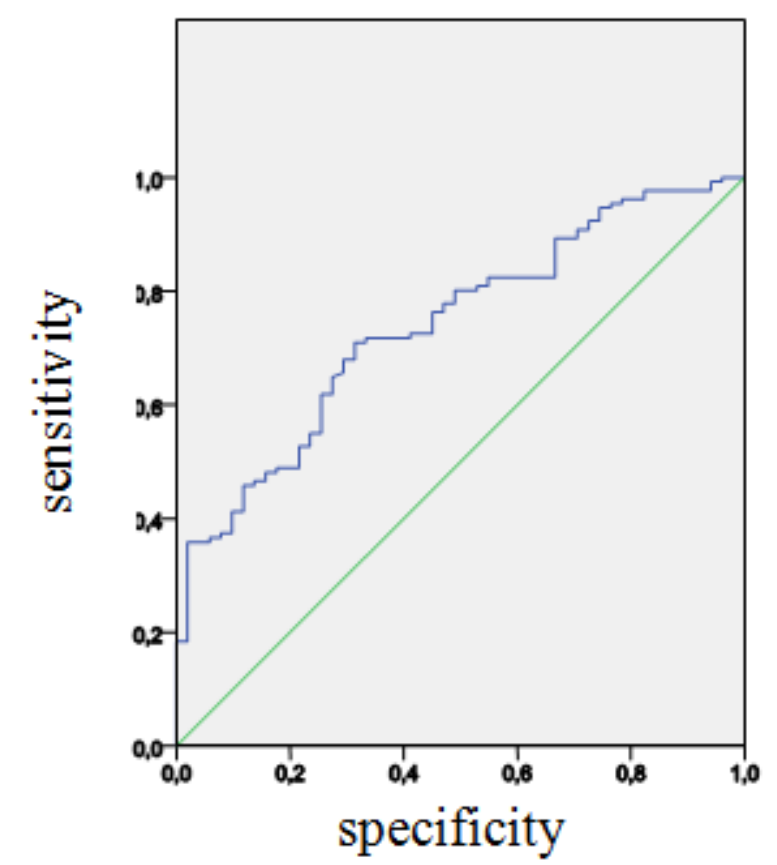

Figure 2. ROC Curve regarding the measurements of netrin

Table 3. Performance Indicators

\begin{tabular}{lll}
\hline indicators & Definitions & Netrin \\
\hline Area under the curve & & 0,739 \\
95\% Confidence Interval & & $0,664-0,814$ \\
Best Cutoff & & $<728.4 \mathrm{pg} / \mathrm{ml}$ \\
Number of Cases & $\mathrm{N}$ & 182 \\
sensitivity & $\mathrm{GP} /(\mathrm{GP}+\mathrm{YN})$ & $93 / 131(\% 71,0)$ \\
specifity & $\mathrm{GN} /(\mathrm{GN}+\mathrm{YP})$ & $35 / 51(\% 68,6)$ \\
PTD & $\mathrm{GP} /(\mathrm{GP}+\mathrm{YP})$ & $93 / 109(\% 85,3)$ \\
NTD & $\mathrm{GN} /(\mathrm{YN}+\mathrm{GN})$ & $35 / 73(\% 47,9)$ \\
accuracy & $(\mathrm{GP}+\mathrm{GN}) /(\mathrm{N})$ & $128 / 182(\% 70,3)$ \\
p value & & $<0,001$ \\
\hline
\end{tabular}

Table 4. Netrin Levels of subgroups

\begin{tabular}{ll}
\hline Groups & Netrin \\
\hline control & $788,8(209,7-2578,8)^{\mathrm{a}, \mathrm{b}}$ \\
hemorrhagic stroke & $666,2(152,3-1944,0)^{\mathrm{a}}$ \\
ischemic stroke & $592,6(40,1-2271,9)^{\mathrm{b}}$ \\
p-value & $<0,001$ \\
\hline
\end{tabular}

a: a statistically significant difference between control and hemorrhagic stroke groups $(\mathrm{p}=0,029)$,

b: a statistically significant difference between control and ischemic stroke groups $(\mathrm{p}<0,001)$.

In the case group of patients who died with patients surviving a median of netrin levels (Table 5) there was no statistically significant difference in terms of $(p=0.782)$. 
Case group with admission NIHSS level of netrin (Table 6 ), there was no statistically significant correlation ( $\mathrm{p}=$ 0.705). Which can be monitored in patients with increased levels of NIHSS at the end of the 6th month of netrin measurements were statistically significantly decreased $(r=-$ $0.455, \mathrm{p}<0.001$ ). Which can be monitored in patients at the end of 6 months compared with baseline NIHSS level increases netrin measurements were statistically significantly decreased $(\mathrm{r}=-0.742, \mathrm{p}<0.001)$.

Types of ischemic stroke cases within the group in terms of media netrin measurements (Table 7) did not show a statistically significant difference $(\mathrm{p}=0.185)$.

Within the group of cases mild, moderate and severe deficits among groups in terms of median netrin measurements (Table 8) showed no statistically significant difference $(p=0.353)$. Within the group of cases mild, moderate, severe deficits among groups in terms of median netrin measurements did not show a statistically significant difference $(\mathrm{p}=0.514)$.

Table 5. Netrin levels according to alive and died patients

\begin{tabular}{ll}
\hline Groups & Netrin \\
\hline alive & $614,4(50,6-1944,0)$ \\
exitus & $529,7(40,1-2271,9)$ \\
p-value & 0,782 \\
\hline
\end{tabular}

Table 6. Correlation Coefficients

\begin{tabular}{llll}
\hline Variables & n & Correlation Coefficient & p-value \\
\hline NIHSS & 131 & $-0,033$ & 0,705 \\
6.th month NIHSS & 107 & $-0,455$ & $<0,001$ \\
Changes in NIHSS & 107 & $-0,742$ & $<0,001$ \\
\hline
\end{tabular}

Table 7. Netrin levels according to the cause of ischemic stroke

\begin{tabular}{ll}
\hline Ischemic stroke & Netrin \\
\hline "1" & $653,4(40,1-2271,9)$ \\
"2" & $726,8(70,4-999,0)$ \\
"3" & $442,9(50,6-1788,1)$ \\
p-value & 0,185 \\
\hline
\end{tabular}

1 large vessel disease (thrombosis or embolism),

2 Cardioembolism,

3. Small vessel disease (lacunal).

Table 8. Netrin levels according to NIHSS levels

\begin{tabular}{ll}
\hline NIHSS & Netrin \\
\hline mild & $592,6(50,6-2271,9)$ \\
moderate & $648,8(122,4-1944,0)$ \\
severe & $496,5(40,1-1177,3)$ \\
p-value & 0,353 \\
mild & $592,6(50,6-2271,9)$ \\
Moderate+severe & $616,7(40,1-1944,0)$ \\
p-value & 0,514 \\
\hline
\end{tabular}

\section{Limitations}

In our study group, we have small number of patients. We have just evaluated the effectiveness of a biomarker, we believe that netrin 1 with other biomarkers of value in prognosis also that should be used. We used NIHSS prognosis evaluation, we did not used other stroke prognosis scales. Netrin is a new marker so due to lack of information in the literature may be limitations in our study.

\section{Discussion}

Developments in the medical treatment of the cause of stroke and a reduction in the incidence of stroke with advancing age, but still affects an expanding population, labor force and causes financial loss. In the management of cerebrovascular disease; as well as preventive treatment of stroke in the early period of medical treatment is of great importance. It is clear that, developments in treatment can be done in the acute phase of stroke is effective in reducing mortality and morbidity. To facilitate the diagnosis in patients with stroke, prognosis and clinical outcomes, early or late evaluate new episodes of stroke or several biomarkers for use in risk stratification is used and continues to be investigated. However stroke patients with clinical examinations for diagnostic imaging methods other than the present routine are not used as a biochemical marker. In addition, patient survival increased with advances in the treatment phase and permanent neurological deficits is intended to minimize the formation. In light of these information, acute stroke and emergency department patients admitted to the serum of human netrin-1 levels of patient survival effect on hemorrhagic and ischemic stroke in patients with serum netrin-1 levels differ between whether, ischemic and hemorrhagic patients, separation of serum netrin 1 level diagnostic value and whether we aimed to observe the prognostic significance. In the cerebrovascular disease, except in the netrin-1 made several studies of different biomarkers, but with netrin-1 is not seen much in the literature to a prospective study.

On the study that Barbieri and his friends carried out with 58 patients in their study the first 24 hours Looking MMP-9, S100 $\beta$, NT pro-BNP and D-dimer patients with NIHSS scores and the patient's clinical condition a significant relationship between has been established (12). Abubakar et al in his study of serum albumin levels of patients in the development of NIHSS scores and 30-day mortality and morbidity effects have been investigated and serum albumin level in mortality and morbidity from the positive effects have been mentioned (13). Wambie et al, which was made in Sweden on population studies indicate that stroke patients with ADMA levels were compared. Of stroke patients and the control group found statistically significant differences (14). In acute ischemic stroke study Cojocaru and his colleagues looked at the first 24 hours, the MPO (Myeloperoxidase) reached significantly higher levels in acute ischemic stroke and are suggested to be used for diagnostic purposes (15). In our study, control, hemorrhagic stroke and ischemic stroke compared levels of netrin media groups, respectively, compared to the control group; hemorrhagic stroke and ischemic stroke group, the median level of netrin statistically significantly lower $(p=0.029$ and $p<0.001)$. Ischemic stroke, 
hemorrhagic stroke compared with the median of the group despite a lower level of netrin difference between the groups was not statistically significant $(p=0.297)$. Within the group of cases of ischemic stroke and media types in terms of netrin measurements observed a statistically no significant difference $(p=0.185)$. Therefore we think that, netrin- 1 in the diagnosis of stroke in the clinic could be used as a diagnostic biomarker.

Netrin proliferation and migration of vascular smooth muscle cells stimulates mitogenic effects such as are known $(16,17)$. Netrin-1; primarily stimulates proliferation in the vessel wall endothelial and vascular smooth muscle cells then increase the adhesion, so as strong mitogen vascular effect. Netrin-1 as well as vascular endothelial growth factor (VEGF) increases (18). This effect of netrin 1 , in the regulation of blood circulation in pathological conditions has been suggested to be therapeutic. In studies, netrin-1, leg ischemia model has been shown to accelerate the formation of new vessels. Again in the rat, model of diabetic neuropathy have been shown to reduce and vasculopathy (9). However, recombinant netrin-1 protein, both functional cardiac infarct formed while increasing the density of vessels (10), netrin-1 in adult rat brain have been shown to contribute to the formation of new vessels (11).

In the Sun and his colleagues study, netrin-1 gene in mice after injection of the first three weeks significantly increased in neurons has been shown that netrin-1. Both endogenous copied and netrin-1 protein were detected in the neurons of the peri-infarct. Peri-infarct area in the early period after the transfer to be in netrin-1 did not reduce infarct size significantly, but the improvement was after stroke engine developing asymmetric.

As a result, studies of netrin-1 in the peri-infarct cortex neurovascular transfer environment and improve motor function after ischemic stroke showed that contribute to the improvement (19). This study shows us that, netrin 1 in the treatment of stroke among treatment options in the future, may be replaced at the same time shed light on the complex effects of netrin 1 provides information on.

Biomarker tracking with the ability to determine prognosis in acute stroke is arranged on a lot of work. In the review conducted in 2008 , according to the results of a multinational study 66; adiponectin, brain natriuretic peptide, C-reactive protein, glial fibrillary acidic protein, glutamate, homocysteine, insulinlik growth factor, intercellular adhesion molecule, matrix metalloproteinase 9, platelet activator inhibitor, prothrombin fragments, soluble tumor necrosis factor receptors 1 , tau, troponin I , troponin $\mathrm{T}$, and a direct relationship between the prognosis is determined by the parameters of thrombomodulin (20).

Abubakar et al., in his study of serum albumin levels of patients on admission NIHSS scores and 30-day mortality and morbidity effects have been investigated and serum albumin level in mortality and morbidity from the positive effects are mentioned (13).

Huang et al., in his study hs-CRP and a significant correlation between 3-month mortality in stroke patients has been established (21).

Hasan et al., in his study, explained that CRP, P-selectin and homocysteine give meaningful information about the 3month morbidity after analyzed the first 24 hours after stroke (22).

Bielewicz et al. in a study conducted in patients NIHSS tau by looking at the value and morbidity of patients 3 months with an inverse relationship between tau values (23).

Jensen et al., in their study of 244 patients with serum osteoprotegerin concentration of stroke patients have established a significant relationship between morbidity and mortality (24).

C. R. Canova in 1999, in his study on 138 stroke cases, he showed that there has no relationship between CRP value and Stroke prognosis (25). When we look at the impact on the prognosis of netrin, although there is no statistically significan correlation between NIHSS level of netrin at the admission of case group ( $p=0,705)$, which can be monitored in patients with increased levels of NIHSS at the end of the 6th month of netrin measurements were statistically significantly decreased $(r=-0,455$ ve $\mathrm{p}<0,001)$. This in stroke patients during application of netrin-1 levels may provide information to us in prospectively followed prognosis suggests.

\section{Result}

When we consider our findings from our study and previous studies on similar issues, we thoughgt that would be useful for serum in patients presenting to the emergency department with stroke netrin-1 levels in the diagnosis of stroke patients and in determining treatment and prognosis of the future.

In this study, we achieved below tips;

1 We believe that, in the diagnosis of stroke patients, Netrin 1 serum levels measured suggest that the diagnosis may be useful as supportive and in our study, the serum of stroke patients netrin-1 levels were determined as low.

2 We are thinking that addmission in stroke patients, serum levels of netrin 1 on patient morbidity gives significant clues. We observed that there is significant relationship between application of netrin-1 levels in patients and NIHSS scores with after 6 months with a change of NIHSS changes.

3 We could not set up meaningfull relationship between strok patients serum netrin-1 levels with patient mortality.

4 We could not make any meaningfull relation between patient netrin-1 level with NIHSS values.

It is clear that there is need for new biomarkers in acute ischemic stroke. In order to determine the prognosis of stroke and mortality in the literature, many markers are used. During our study, diagnosis of stroke patients have low levels of netrin-1. According to the type of stroke, there is no difference between the levels of netrin-1. Netrin-1 levels were not associated with mortality. 


\section{References}

[1] World Health Organisation. Cerebrovascular Disorders (Offset Publications). Geneva: World Health Organization; ISBN 9241700432. OCLC 4757533. 1978.

[2] Goldstein LB, Samsa GP. Reliability of the National Institutes of Health Stroke Scale. Extension to non-neurologists in the context of a clinical trial. Stroke. 1997; 28(2):307-10.

[3] Kothari RU, Brott T, Broderick JP, et al. Emergency physicians. Accuracy in the diagnosis of stroke. Stroke. 1995; 26(12):2238-41.

[4] Shafqat S, Kvedar JC, Guanci MM, et al. Role for telemedicine in acute stroke: feasibility and reliability of remote administration of the NIH Stroke Scale. Stroke. 1999; 30:2141-2145.

[5] Freitas C, Larrivee, B, Eichmann A. Netrins and UNC5 receptors in angiogenesis. Angiogenesis 2008; 11, 23-29.

[6] Basnakian AG. Netrin-1: A potential universal biomarker for acute kidney injury. Article in Press Am J Physiol Renal Physiol. 2008.

[7] Huber AB, Kolodkin AL, Ginty DD, et al. Signaling at the growth cone: ligand-receptor complexes and the control of axon growth and guidance. Annu Rev Neurosci 2003, 26:509563.

[8] Serafini, T., Colamarino, S.A., Leonardo ED, et al. Netrin-1 is required for commissural axon guidance in the developing vertebrate nervous system. Cell, 1996; 87, 1001-1014

[9] Wilson BD, Li M, Park KW, et al. Netrins promote developmental and therapeutic angiogenesis. Science, 2006; 313, 640-644.

[10] Ahmed RP, Haider KH, Shujia J, et al.2010. Sonic Hedgehog gene delivery to the rodent heart promotes angiogenesis via iNOS/netrin-1/PKC pathway. PLoS One 5, e8576.

[11] Fan, Y., Shen, F., Chen, Y., Hao, Q., Liu, W., Su, H., Young, W.L., Yang, G.Y., 2008. Overexpression of netrin-1 induces neovascularization in the adult mouse brain. J. Cereb. Blood Flow Metab. 28, 1543-1551.

[12] Barbieri A, Giuliani E, Carone C, Pederzoli F, Mascheroni G, Greco G, Stucchi C, Genedani S. Clinical severity of ischemic stroke and neural damage biomarkers In the acute setting: The STROke-MArkers (STROMA) Study. Minerva Anestesiol. 2013 Mar 19.

[13] Abubakar S, Sabir A, Ndakotsu M, Imam M, Tasiu M. Low admission serum albumin as prognostic determinant of 30-day case fatality and adverse functional outcome following acute ischemic stroke. Pan Afr Med J. 2013; 14:53. doi: 10.11604/pamj.2013.14.53.1941. Epub 2013 Feb 7.

[14] Wanby P, Teerlink T, Brudin L, Brattstr"om L, Nilsson I, Palmqvist P, Carlsson M. (2006). Asymmetric dimethylarginine (ADMA) as a risk marker for stroke and TIA in a Swedish population. Atherosclerosi, 185:271-277.
[15] The National Institute of Neurological Disorders and Stroke rt-PA Stroke Study Group. Tissue plasminogen activator for acute ischemic stroke. N Engl J Med. 1995; 333: 15811587.73) Hacke W, Kaste M, Fieschi C, Toni D, Lesaffre E, von Kummer R, Boysen G, Bluhmki E, Hoxter G, Mahagne $\mathrm{MH}$, et al. Intravenous thrombolysis with recombinant tissue plasminogen activator for acute hemispheric stroke: the European Cooperative Acute Stroke Study (ECASS). JAMA. 1995; 274: 1017-1025

[16] Nakashiba T, Ikeda T, Nishimura $S$, Tashiro K, Honjo T, Culotti JG, Itohara S: Netrin-G1: a novel glycosyl phosphatidylinositol-linked mammalian netrin that is functionally divergent from classical netrins. J Neurosci 2000, 20:6540-6550.

[17] Serafini T, Kennedy TE, Galko MJ, Mirzayan C, Jessell TM, Tessier-Lavigne M: The netrins define a family of axon outgrowth-promoting proteins homologous to C. elegans UNC-6. Cell 1994, 78:409-424.

[18] Park, K.W., Crouse, D., Lee, M., Karnik, S.K., Sorensen, L.K., Murphy, K.J., Kuo, C.J., Li, D.Y., 2004. The axonal attractant Netrin-1 is an angiogenic factor. Proc. Natl. Acad. Sci. U. S. A. $101,16210-16215$.

[19] Sun H, Le T, Chang TT, Habib A, Wu S, Shen F, Young WL, $\mathrm{Su} \mathrm{H}$, Liu J. AAV-mediated netrin-1 overexpression increases peri-infarct blood vessel density and improves motor function recovery after experimental stroke. Neurobiol Dis. 2011 Oct; 44(1):73-83. doi: 10.1016/j.nbd.2011.06.006. Epub 2011 Jun 25 .

[20] Clark WM, Wissman S, Albers GW, Jhamandas JH, Madden $\mathrm{KP}$, Hamilton S. Recombinant tissue-type plasminogen activator (Alteplase) for ischemic stroke 3 to 5 hours after symptom onset. The ATLANTIS Study: a randomized controlled trial. Alteplase 53 Thrombolysis for Acute Noninterventional Therapy in Ischemic Stroke. JAMA. 1999; 282:2019-26.

[21] Huang Y, Jing J, Zhao XQ, Wang CX, Wang YL, Liu GF, Wang CJ, Liu LP, Yang XM, Jiao Y, Jiao Y, Wang LS, Wang YJ, Gu WK. High-sensitivity C-reactive protein is a strong risk factor for death after acute ischemic stroke among Chinese. CNS Neurosci Ther. 2012 Mar; 18(3):261-6.

[22] Hasan N, McColgan P, Bentley P, Edwards RJ, Sharma P. Towards the identification of blood biomarkers for acute stroke in humans: a comprehensive systematic review. Br J Clin Pharmacol. 2012 Aug; 74(2):230-40.

[23] Bielewicz J, Kurzepa J, Czekajska-Chehab E, Stelmasiak Z, Bartosik-Psujek H. Does serum Tau protein predict the outcome of patients with ischemic stroke? J Mol Neurosci. 2011 Mar; 43(3):241-5. doi: 10.1007/s12031-010-9403-4. Epub 2010 Jun 12.

[24] Jensen JK, Ueland T, Atar D, Gullestad L, Mickley H, Aukrust P, Januzzi JL. Osteoprotegerin concentrations and prognosis in acute ischaemic stroke. J Intern Med. 2010 Apr;267(4):410-7. doi: 10.1111/j.1365-2796.2009.02163.x. Epub 2009 Aug 26.

[25] C.R. Canova, C. Courtin, W.H. Reinhart. C-reactive protein (CRP) in cerebrovascular events. Atherosclerosis 1999; 147(1):49-53. 